\title{
Development and application of a novel ELISA for detecting antibodies against group I fowl adenoviruses
}

\author{
Qing Pan • Jing Wang • Yulong Gao ${ }^{1}$. Hongyu Cui ${ }^{1}$. Changjun Liu ${ }^{1} \cdot$ Xiaole Qi ${ }^{1} \cdot$ Yanping Zhang ${ }^{1}$. \\ Yongqiang Wang ${ }^{1} \cdot \mathrm{Kai}^{\mathrm{Li}}{ }^{1} \cdot \mathrm{Li} \mathrm{GaO}^{1} \cdot$ Xiaomei Wang ${ }^{1,2}$
}

Received: 8 August 2019 / Revised: 11 October 2019 / Accepted: 19 October 2019 / Published online: 14 December 2019

(C) Springer-Verlag GmbH Germany, part of Springer Nature 2019

\begin{abstract}
Since 2015, outbreaks of hepatitis-hydropericardium syndrome (HPS) caused by a novel genotype of fowl adenovirus 4 (FAdV4) infection have created serious economic losses in China. Given that other serotypes of hypervirulent FAdVs have also been reported in poultry around the world, a common ELISA for all serotypes within the group I fowl adenoviruses (FAdV-I) is urgently needed, especially for clinical epidemic serotypes. In this study, we used high purity and concentration virions of FAdV4 and developed a common ELISA for detecting antibodies against 12 FAdV-I serotypes. The developed ELISA was able to distinguish between antibodies against FAdV-I, FAdV-III, and other heterologous viruses without any cross-reaction. Furthermore, the ELISA showed higher sensitivity than the FAdV-1-based ELISA to the novel FAdV-4 found in China. Moreover, since there are no commercial vaccines against FAdVs in China, the ELISA was applied to detect sera samples from specific pathogen-free chickens inoculated with inactivated FAdV-1, FAdV-4, and FAdV-8a. The assay showed high sensitivities for all three detected serotypes within FAdV-I. In conclusion, a novel, common ELISA for FAdV-I was developed in this study and could be a powerful tool for seroepidemiological investigations and FAdVs vaccine development.
\end{abstract}

Keywords Fowl adenovirus $\cdot$ ELISA $\cdot$ Group-specific $\cdot$ FAdV-4 $\cdot$ Antibody detection

\section{Introduction}

Fowl adenoviruses are divided into three groups, group I fowl adenovirus (FAdV-I) contains five species (A to E) with 12 serotypes (1-8a, 8b-11) (Hess 2000) isolated from fowls, group II (FAdV-II) includes the hemorrhagic enteritis virus (HEV) of turkeys and the marble spleen disease virus (MSDV) of pheasants (Domermuth et al. 1980), and group III (FAdV-III) is mainly associated with egg drop syndrome virus (EDSV) (Huang et al. 2015). Inclusion body hepatitis (IBH), hydropericardium syndrome (HPS), and gizzard

Qing Pan and Jing Wang contributed equally to this work.

Xiaomei Wang

wangxiaomei@caas.cn

1 Division of Avian Infectious Diseases, State Key Laboratory of Veterinary Biotechnology, Harbin Veterinary Research Institute, Chinese Academy of Agricultural Sciences, Harbin 150001, China

2 Jiangsu Co-innovation Center for the Prevention and Control of Important Animal Infectious Disease and Zoonose, Yangzhou University, Yangzhou 225009, China erosion (GE) associated with FAdV-I infection have been reported worldwide, e.g., in Pakistan (Khawaja et al. 1988; Mansoor et al. 2009), Chile (Toro et al. 1999), Korea (Choi et al. 2012), Canada (Dar et al. 2012), Hungary (Kaján et al. 2013), India (Mittal et al. 2014), Japan (Mase and Nakamura 2014), South Africa (Joubert et al. 2014), Mexico (VeraHernández et al. 2016), Poland (Niczyporuk 2016), and China (Zhao et al. 2015), resulting in significant economic losses to the poultry industry. HPS is caused by FAdV-4 (Pan et al. 2017a), whereas IBH is always related to FAdV2, FAdV-11, FAdV-8a, or FAdV-8b (Morshed et al. 2017) and GE is mainly caused by FAdV-1 (Matczuk et al. 2017). Notably, severe HPS, with a high mortality rate of $20-80 \%$, caused by a novel genotype FAdV-4 has been widespread in China since 2015 ( $\mathrm{Li}$ et al. 2016; Pan et al. 2017a) and is a major threat to the poultry industry.

In recent years, a variety of FAdVs detection methods have been developed for large-scale seroepidemiological investigations and HPS vaccine evaluation. For antigen detection, polymerase chain reaction (PCR) (Günes et al. 2012), quantitative PCR (qPCR) (Pan et al. 2017c), high-resolution melting (HRM) curve analysis (Steer et al. 2009), loop-mediated 
isothermal amplification (Yuan et al. 2019; Zhai et al. 2019), and sandwich enzyme-linked immunosorbent assays (ELISA) (Shao et al. 2019a; Shao et al. 2019b) have been developed. The traditional methods for FAdVs serological diagnosis are agar gel precipitation (AGPT) and virus neutralization (VN) tests. While AGPT is generally less sensitive, the process of $\mathrm{VN}$ is not conducive to rapid and large-scale diagnosis. Thus, a simple, rapid, and sensitive diagnostic method is urgently required for FAdVs detection, for which ELISA, which is widely used in large-scale serological investigation, is simple to operate, and has high sensitivity, is an excellent candidate technology.

Prevention and control of HPS have been attempted, mainly through the use of inactivated virus (Meng et al. 2019; Pan et al. 2017b) or subunit vaccines (Ruan et al. 2018; Schachner et al. 2014; Shah et al. 2017; Wang et al. 2018), for some FAdV serotypes. The immune response to vaccines is generally monitored by the presence of an antigen-specific antibody and the neutralization antibody. In one recent study, recombinant fiber-based indirect ELISA was used to detect serum samples from chickens experimentally inoculated with different FAdV-1 or FAdV-4 strains (Feichtner et al. 2018). A recombinant hexon-based single serum dilution ELISA was also developed to measure the hexon-specific antibodies against FAdV-4 in sera of chickens (Rajasekhar and Roy 2014). Both of the ELISAs mentioned above were FAdV serotypespecific and based on variable recombinant proteins. However, some other FAdV serotypes, such as FAdV-1 and FAdV-8a, have also been shown to cause serious economic losses to the poultry industry.

Unfortunately, there is currently no commercial FAdV-I ELISA kit in China, thus a common ELISA for all FAdV-I serotypes is urgently needed. In this study, we developed a group-specific and sensitive ELISA based on the novel genotype of FAdV-4 for detecting antibodies against twelve FAdVI serotypes. The common ELISA provides a powerful tool for the seroepidemiological investigations and vaccine development.

\section{Materials and methods}

\section{Viruses and cells}

As we reported previously (Pan et al. 2018), the HLJFAd15 strain (GenBank no. KU991797) was isolated from layers and identified as serotype 4 (FAdV-4). The FAdV-1 CELO (GenBank No. U46933) and FAdV-8a (a clinical isolate) strains were deposited at the State Key Laboratory of Veterinary Biotechnology, Harbin Veterinary Research Institute (Harbin, China). Chicken leghorn male hepatocellular (LMH) cells were kindly gifted by Prof. Guozhong Zhang (China Agricultural University, Beijing, China) and were cultured in Dulbecco's Modified Eagle's Medium (DMEM) (Thermo Fisher Scientific, Waltham, MA, USA) supplemented with $10 \%$ fetal bovine serum (FBS) (Gibco, San Diego, CA, USA), $100 \mathrm{IU} / \mathrm{ml}$ penicillin, and $100 \mu \mathrm{g} / \mathrm{ml}$ streptomycin.

\section{Propagation and purification of viruses}

Following HLJFAd15 (FAdV-4) strain propagation and purification, the FBS concentration in confluent cultures was reduced to $2 \%$ for maintenance. The LMH cells were infected with 0.1 multiplicity of infection (MOI) of FAdV-4 and incubated at $37{ }^{\circ} \mathrm{C}$ in a $5 \% \mathrm{CO}_{2}$ atmosphere for 3 days. Formaldehyde was added to the culture medium for virus inactivation, and viral particles were harvested from virusinfected LMH cells at a concentration of $0.2 \%$ in the final product (Kim et al. 2014). Culture fluids were collected and, after 3 cycles of freezing and thawing, the mixture was centrifuged at $5000 \times \mathrm{g}$ for $15 \mathrm{~min}$ to remove cellular debris. Supernatants were transferred to $20 \%, 30 \%(\mathrm{w} / \mathrm{w})$ sucrose solution and centrifuged at 30,000 rpm for $3 \mathrm{~h}$ using a Beckman SW 32 rotor in a Model Optima XPN-100 ultracentrifuge (Beckman Coulter, Brea, CA, USA). Virus pellets were collected and suspended in phosphate-buffered saline (PBS). Culture suspensions were collected, purified in $1.33 \mathrm{~g} / \mathrm{ml}$ Cesium Chloride $\left(\mathrm{CsCl}_{2}\right)$ (Amresco, Solon, USA), and centrifuged at 35,000 rpm for $16 \mathrm{~h}$ using a Beckman SW 55 rotor. Two discrete bands were formed following final ultracentrifugation. The bands were aspirated with a syringe by puncturing the side of the tube, suspended in PBS, centrifuged at 32,000 rpm for $2 \mathrm{~h}$ using a Beckman SW 32 rotor, and culture fluids were collected (Pan et al. 2018). The morphology of FAdV-4 preparations was verified by electron microscopy.

\section{Development and optimization of ELISA}

Carbonate buffer ( $\mathrm{pH}=9.6)$, tris- $\mathrm{HCl}$ buffer $(\mathrm{pH}=8.0)$, and phosphate buffer $(\mathrm{pH}=7.4)$ were used as coating buffers. PBST containing 5\% skim milk, PBS containing 5\% bovine serum, and PBS containing 5\% gelatin were used as blocking buffers. FAdV-4 stocks with a concentration of $1.03 \mathrm{mg} / \mathrm{ml}$, as measured by micro-volume spectrophotometer (Implen, Munchen, Germany), were obtained and prepared into working dilutions $(1 \mu \mathrm{g} / \mathrm{ml}, 3 \mu \mathrm{g} / \mathrm{ml}$, and $5 \mu \mathrm{g} / \mathrm{ml})$ using coating buffer. The working dilutions were added into microtitre plates $(100 \mu \mathrm{l} /$ well $)$ and incubated at $4{ }^{\circ} \mathrm{C}$ for 12,16 , or 20 h. After incubation with the coating antigen, the plates were washed three times with PBS containing $0.05 \%$ Tween-20 (PBST) and then incubated with blocking solution at $37{ }^{\circ} \mathrm{C}$ for 1,2 , or $3 \mathrm{~h}$. After three washes, serum samples were diluted $1: 25,1: 50,1: 100,1: 200,1: 400,1: 800,1: 1,600$, and 1:3200 and incubated at $37{ }^{\circ} \mathrm{C}$ for $0.5,1$, and $2 \mathrm{~h}$, respectively. Following incubation, samples were washed three times and incubated at $37^{\circ} \mathrm{C}$ for $0.5,1$, and $2 \mathrm{~h}$ with HRP-conjugated 
rabbit anti-mouse antibodies (Sigma, Missouri, USA) diluted $1: 2500,1: 5000$, and 1:10,000, respectively. After washing, $100 \mu \mathrm{l}$ tetramethylbenzidine (TMB) substrate (Amresco, Solon, USA) was added to each well and the plates were incubated in the dark for 5, 8, and $11 \mathrm{~min}$. The enzymatic reaction was quenched by hydrofluoric acid and the optical density (OD) was determined at $630 \mathrm{~nm}$. ODs presented represent the mean from duplicate wells. The optimal conditions were determined by evaluating the OD values and the positive/negative ratio $(\mathrm{P} / \mathrm{N})$ of the samples. The cut-off was determined according to the sample/positive $(\mathrm{S} / \mathrm{P})$ ratio by calculating the arithmetic mean plus three times the standard deviation (SD).

\section{Specificity and sensitivity of the common ELISA}

Different serotypes of FAdV-I (FAdV-1, FAdV-4 GY, FAdV10, FAdV-9, FAdV-11, FAdV-8a) positive serum were kindly provided by Dr. Junping Li (China Institute of Veterinary Drug Control (IVDC), Beijing, China); EDSV (FAdVIII) positive serum was purchased from IVDC; H5 AIV, H9 AIV, NDV, IBV, and ILTV positive serum was kindly gifted by Prof. Yunfeng Wang (Harbin Guosheng Biotechnology Co., Ltd, Harbin, China). Sensitivity of the common ELISA was assessed with FAdV-4 positive sera diluted at a gradient of 1:100, 1:200, $1: 400,1: 800,1: 1600,1: 3200,1: 6400,1: 12,800,1: 25,600$, and $1: 51,200$. The specificity and sensitivity of the assay were conducted according to the cut-off titer value in three independent replications.

\section{Experimentally recruited serum samples}

Forty specific antibody-negative chickens were divided into four groups: FAdV-1 immunization group ( $n=10)$, FAdV-4 immunization group ( $n=10)$, FAdV-8a immunization group $(n=10)$, and a control group of age matched unvaccinated chickens $(n=10)$. The formaldehyde inactivated antigen solution was emulsified with oil adjuvant at a ratio of 25:75 $(\mathrm{w} / \mathrm{w})$. The immunization group was immunized intramuscularly with $0.5 \mathrm{ml}$ inactivated viruses containing $10^{6} \mathrm{TCID}_{50}$ FAdV-1, FAdV-4, FAdV-8a antigens per chicken at the age of 21 days. Five serum samples of chickens in each group were collected weekly post inoculation until the termination of the experiment.

\section{Screening of field sera}

In total, 850 clinical serum samples were examined by the developed ELISA for the presence of FAdV antibodies. These samples were collected from breeder flocks of unknown disease and vaccination status in Jiangsu, Heilongjiang province. FAdV antibodies were detected by the common ELISA and results were compared with a commercial kit (BioChek, Reeuwijk, The Netherlands) for FAdV-I. Anti-FAdVs antibodies were detected by indirect immunofluorescence assay (IFA).

\section{Indirect immunofluorescence assay}

An IFA was carried out on LMH cells plated in 24 well plates and infected with $0.1 \mathrm{MOI}$ of the FAdV-4. After 2-day incubation at $37{ }^{\circ} \mathrm{C}$ in a $5 \% \mathrm{CO}_{2}$ atmosphere, the wells were fixed with iced ethanol for $20 \mathrm{~min}$ at room temperature and washed three times with PBS. Clinical serum samples diluted $1: 10$ in PBS were used as primary antibodies for $1 \mathrm{~h}$ at $37^{\circ} \mathrm{C}$. After washing three times, Alexa Fluor ${ }^{\mathrm{TM}} 488$ goat anti-mouse $\operatorname{IgG}(\mathrm{H}+\mathrm{L})$ secondary antibody (1:200) (Invivogen, San Diego, California, USA) was added, followed by incubation for $1 \mathrm{~h}$ at $37{ }^{\circ} \mathrm{C}$. After washing three times, the wells were investigated by EVOS F1 inverted fluorescence microscope.

\section{Statistical analysis}

Differences between the two groups were evaluated by Student's $t$ test and considered significant at $* * p<0.01$.

\section{Results}

\section{Propagation and purification of viruses}

Purified intact virus particles were used as an optimal coating antigen to develop a common ELISA in this study. Highpurity virions were collected by sucrose and $\mathrm{CsCl}_{2}$ density gradient centrifugation. Subsequently, FAdV-4 stocks with a concentration of $1.03 \mathrm{mg} / \mathrm{ml}$, as measured by micro-volume spectrophotometer, were obtained. The FAdV-4 virions were verified by electron microscopy, with negatively stained preparations showing nearly round surface with a diameter of 70 $90 \mathrm{~nm}$. As shown in Fig.1, the purified virions were intact and of high purity, and the preparation had good dispersion and was evenly distributed throughout the field.

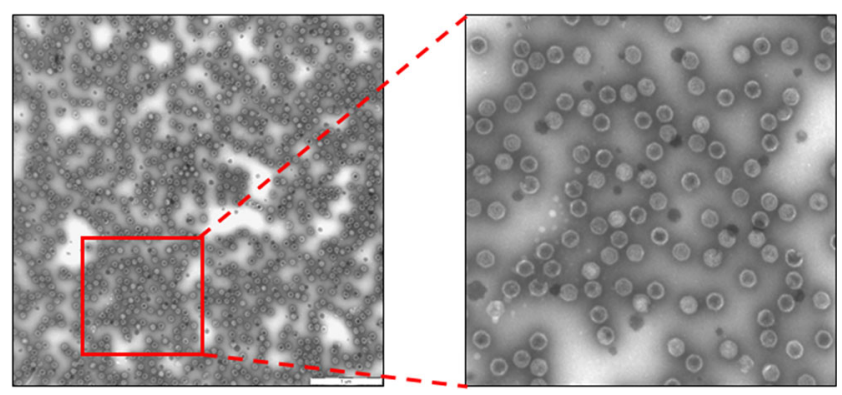

Fig. 1 The morphology of the purified virus particles from cell-cultured HLJFAd15 (GenBank no. KU991797) by electron microscopy 


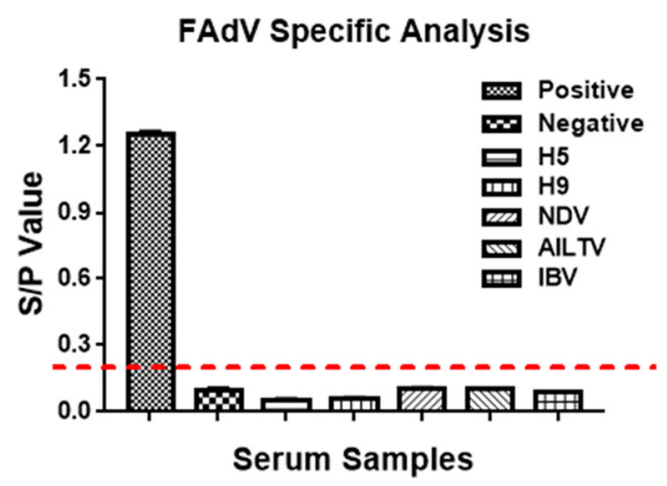

Fig. 2 FAdV-specificity assay for the common ELISA using different heterologous viruses, including H5 and H9 AIV, NDV, IBV, ILTV. SPF chicken serum as negative control; FAdV-4 positive serum as positive control

\section{Development and optimization of ELISA}

The optimum working range of antigen and serum were decided by checkerboard titration and determined by the greatest $\mathrm{P} / \mathrm{N}$ ratio. Antigen concentrations of $3 \mu \mathrm{g} / \mathrm{ml}$ were added to microtitre plates coated with $100 \mu \mathrm{l}$ in carbonate buffer ( $\mathrm{pH}$ 9.6). Following incubation for $16 \mathrm{~h}$ at $4{ }^{\circ} \mathrm{C}$, the plates were washed three times with PBST and blocked with PBST containing 5\% non-fat milk for $2 \mathrm{~h}$ at $37{ }^{\circ} \mathrm{C}$. Serum samples diluted 1:100 in PBS were incubated for $30 \mathrm{~min}$ at $37{ }^{\circ} \mathrm{C}$. After washing three times, secondary antibodies were incubated for $30 \mathrm{~min}$ at $37{ }^{\circ} \mathrm{C}$ before a final washing step. Subsequently, $100 \mu \mathrm{TMB}$ substrate was added to each well and the plates were incubated in the dark for 5 min. The enzymatic reaction was quenched with $100 \mu 10.5 \mathrm{M}$ hydrofluoric acid and the OD values were determined at $630 \mathrm{~nm}$. Based on the arithmetic mean S/ $P$ value of the 200 clinical negative samples (0.047) and the SD value (0.051), the cut-off for the ELISA was determined to be 0.199 . Serum samples with $\mathrm{S} / \mathrm{P}$ value greater or less than 0.199 were determined to be positive or negative, respectively.

\section{ELISA specificity}

In the specificity assay, the negative control serum collected from non-treated SPF chickens was confirmed to be negative by the common ELISA developed in this study, and the positive control serum obtained from SPF chickens inoculated with HLJFAd15 was detected to be positive. As shown in Fig. 2, the S/P values of other heterologous viruses, including H5 and H9 AIV, NDV, IBV, and ILTV positive sera, were below the established cut-off value (ranging from 0.056 to 0.106 ) and were hence determined to be negative by the developed ELISA. In group-specificity and serotype-specificity assays, EDSV (FAdV-III)-positive serum tested by the common ELISA was negative (Fig. 3a). Different serotypes of FAdV-I including FAdV-1, FAdV-4 GY, FAdV-8a, FAdV-9, FAdV-10, and FAdV-11 positive serums ranged from 0.456 to 1.185 (Fig. 3b), above the ELISA cut-off value, were determined to be positive.

\section{FAdV-4 ELISA and FAdV-1 ELISA sensitivity comparison}

Sensitivity analyses comparing the FAdV-4 ELISA and FAdV-1 ELISA using serum samples collected from chickens infected with HPS showed a significantly higher sensitivity of the ELISA developed based on FAdV-4 in this study than the FAdV-1-based ELISA. As shown in Fig. 4, the limit of detection (LOD) of the common ELISA developed for HLJFAd15 (FAdV-4) positive sera was 1:25,600 while the ELISA based on FAdV-1 was 1:6400. The common ELISA showed higher $\mathrm{S} / \mathrm{P}$ values than the FAdV-1-based ELISA detecting antibodies against FAdV-4 positive sera with each dilution assayed.

\section{Kinetics of antibody response after FAdV-1, FAdV-4, and FAdV-8a inoculation}

Kinetic analyses of specific antibody responses following inoculation of all three FADV hypervirulent serotypes were assessed by the common ELISA. FAdV-I antibodies in the

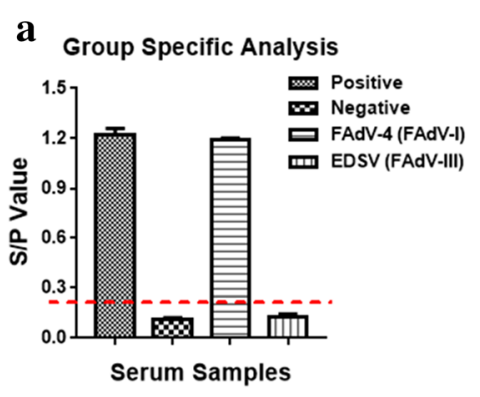

Fig. 3 Group-specificity and serotype-specificity assay for the common ELISA using EDSV (FAdV-III) (a) and different serotypes of FAdV-I, including FAdV-1, FAdV-4 GY, FAdV-10, FAdV-9, FAdV-11, and FAdV-

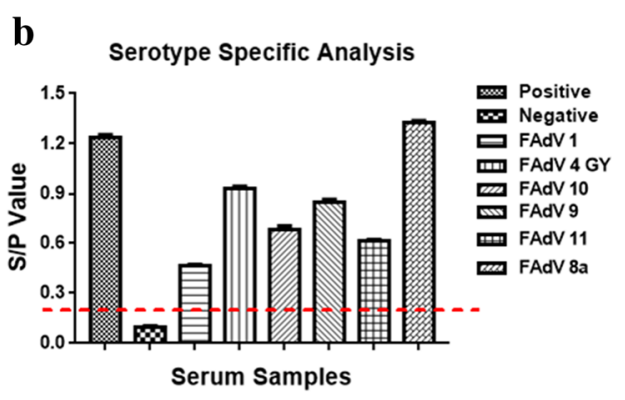

8a, respectively (b). SPF chicken serum as negative control; FAdV-4 positive serum as positive control 


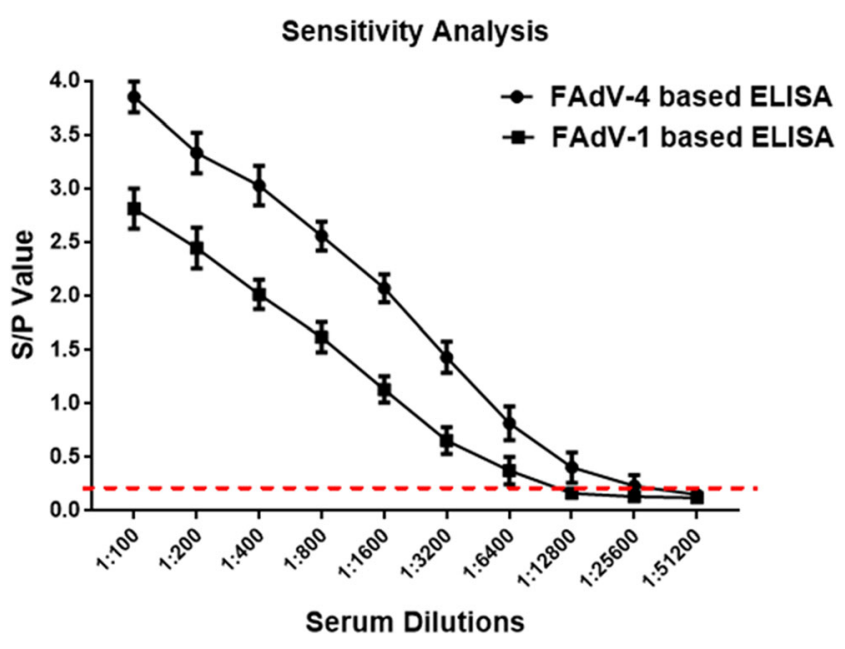

Fig. 4 Diagrammatic representation of cut-off baseline with different positive serum samples. The dotted line represents the cut-off line and the solid line represents FAdV-4 positive serum samples with different antibody titres detected by FAdV-1- and FAdV-4-based ELISA, respectively

negative control group were all negative when tested by the common ELISA. As shown in Fig. 5, serum antibody titres of the experimentally inoculated chickens increased with the time of immunization. In chickens inoculated with inactivated FAdV-1 (Fig. 5a), FAdV-4 (Fig. 5b), or FAdV-8a (Fig. 5c), the earliest detection of antibodies occurred 2 weeks after inoculation $(p<0.01)$. Two weeks after immunization with inactivated virus, the positive rate of antibodies against all three detected serotypes within FAdV-I reached $100 \%$. The antibodies against the emergent novel FAdV-4 showed higher $\mathrm{S} / \mathrm{P}$ values than antibodies against FAdV-1 and FAdV-8a.

\section{Field serum screening}

In field sera, the common ELISA detected 69.1\% (587/850) positives, whereas the commercial FAdV-I ELISA kit test detected $72.2 \%(614 / 850)$ positives (Table 1$)$. The positive coincidence rate between the commercial kit and the developed ELISA was $92.99 \%$ and the negative coincidence was
Table 1 Coincidence assay between a commercial ELISA kit (BioChek) and the common ELISA developed in this study

\begin{tabular}{llll}
\hline & Positive & Negative & Total \\
\hline Commercial ELISA kit & 614 & 236 & 850 \\
Common ELISA & 587 & 263 & 850 \\
Coincidence & $93.49 \%$ & $94.49 \%$ & $93.76 \%$ \\
\hline
\end{tabular}

$89.92 \%$; the total coincidence was $92.43 \%$. Anti-FAdVs antibodies were detected by IFA. The common ELISA and IFA detected $71.3 \%(107 / 150)$ and $71.3 \%$ (107/150) positives, respectively, and the total coincidence between the common ELISA and IFA was $100 \%$ (data not shown).

\section{Discussion}

HPS induced by FAdV-4 has emerged across several different areas in China since 2015 (Niu et al. 2018; Zhao et al. 2015) and caused serious economic losses and poses a great threat to the poultry industry. The pathogenic FAdV-4 strain has been isolated and characterized as a novel genotype (Ye et al. 2016). However, there are currently no commercial FAdV-I or FAdV-4 ELISA kits in China for detection of antibodies against the pathogenic FAdVs or FAdV-4 specifically. Many studies have been conducted on FAdVs antibody detection using ELISA based on FAdV-1. In one study, CELO (FAdV-1) virus was used as a coating antigen to establish an ELISA method for detecting FAdV-1 and avian adenovirus-associated virus (Dawson et al. 1980). As reported, FAdV-4 showed higher cross-reactivity among 10 serotypes than did FAdV-1 (Calnek et al. 1982). Given that homologous ELISA reactions were stronger than heterologous reactions in many cases, we evaluated FAdV-4 as a coating antigen to develop a common ELISA for detecting all twelve FAdV-I serotypes. The ELISA developed based on FAdV-4 in this study showed considerable cross-reaction within the twelve FAdV-I serotypes and higher sensitivity than the

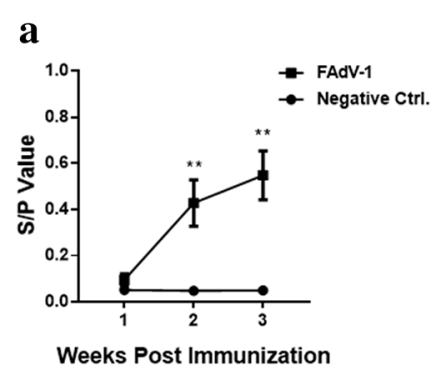

Fig. 5 Mean $\mathrm{S} / \mathrm{P}$ values of serum samples collected weekly after intramuscular inoculation with $0.5 \mathrm{ml}$ inactivated FAdV-1 (a), FAdV-4 (b), and FAdV-8a (c) viruses and age matched unvaccinated chickens
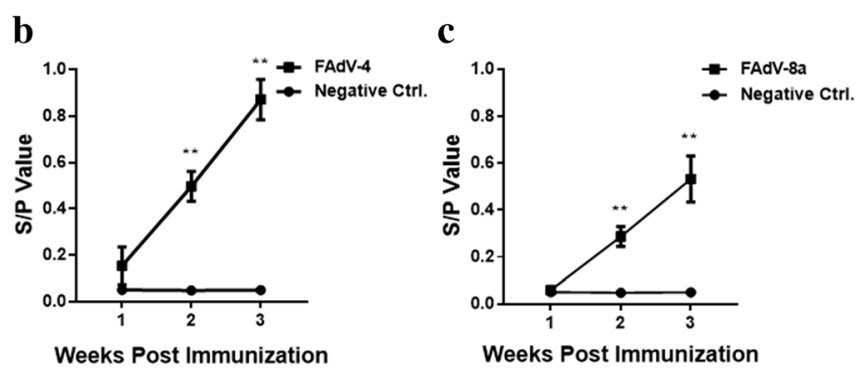

(Negative Ctrl.) evaluated by the common ELISA. The S/P value of the serum samples greater or less than 0.199 were determined to be positive or negative, respectively 
FAdV-1-based ELISA for detecting antibodies against the novel emergent FAdV-4. Ultimately, we successfully established a common ELISA for specifically detecting antibodies against all twelve FAdV-I serotypes, which could be a powerful tool for seroepidemiological investigation of IBH, GE, and, especially, the emergent HPS in China.

Unfortunately, there is no commercial vaccine against FAdVs, including the FAdV-4 strain, and a common detection method is urgently needed to facilitate vaccine development and evaluation. Though several recombinant protein-based ELISAs have been used to detect antibodies in chickens inoculated with subunit vaccines, such as rFiber-1, rFiber-2, and rHexon protein, these ELISA were able to detect antibodies against FAdV-4-specific (Feichtner et al. 2018; He et al. 2018; Rai et al. 2005; Rajasekhar and Roy 2014). Furthermore, prokaryotic expressed proteins need to be renatured to restore tertiary and quaternary spatial structures, which may lead to false-negative results from assays using recombinant proteins. Considering that FAdV-4 and some other serotypes within FAdV-I are pathogenic to chickens worldwide (Grgić et al. 2011; Lim et al. 2011; Morshed et al. 2017), a common ELISA should be more suitable for FAdVs vaccine development. The common ELISA developed in our study was applied to detect serum samples from SPF chickens inoculated with inactivated FAdV-1, FAdV-4, and FAdV-8a, and showed high sensitivity for all three FAdV hypervirulent serotypes. These results suggest that the common ELISA developed in our study is capable of monitoring antibodies against all serotypes in variable FAdV-I vaccine development and evaluation.

In conclusion, the common ELISA developed in this study showed considerable cross-reactivity among all 12 FAdV serotypes and was capable of detecting specific antibodies against FAdV-I. Furthermore, the ELISA showed higher sensitivity in detecting serum samples of HPS caused by the novel FAdV-4 genotype that has recently emerged in China. Our ELISA could be a powerful tool for seroepidemiological investigations and development of vaccines for different FAdV-I serotypes.

Funding information This work was partly supported by the National Key R\&D Program (2016YFD0500800), National Natural Science Foundation of China (31602073), and the China Agriculture Research System (CARS-41-G15).

\section{Compliance with ethical standards}

Conflict of interest The authors declare that they have no competing interests.

Ethics statement The animal experiments were approved by the Animal Care and Use Committee of Harbin Veterinary Research Institute (Harbin, China) and performed in accordance with the 'Guidelines for Experimental Animals' of the Ministry of Science and Technology (Beijing, China).

\section{References}

Calnek BW, Shek WR, Menendez NA, Stiube P (1982) Serological crossreactivity of avian adenovirus serotypes in an enzyme-linked immunosorbent assay. Avian Dis 26(4):897-906

Choi KS, Kye SJ, Kim JY, Jeon WJ, Lee EK, Park KY, Sung HW (2012) Epidemiological investigation of outbreaks of fowl adenovirus infection in commercial chickens in Korea. Poult Sci 91(10):2502-2506

Dar A, Gomis S, Shirley I, Mutwiri G, Brownlie R, Potter A, Gerdts V, Tikoo SK (2012) Pathotypic and molecular characterization of a fowl adenovirus associated with inclusion body hepatitis in Saskatchewan chickens. Avian Dis 56(1):73-81

Dawson GJ, Orsi LN, Yates VJ, Chang PW, Pronovost AD (1980) An enzyme-linked immunosorbent assay for detection of antibodies to avian adenovirus and avian adenovirus-associated virus in chickens. Avian Dis 24(2):393-402

Domermuth CH, Weston CR, Cowen BS, Colwell WM, Gross WB, Dubose RT (1980) Incidence and distribution of "avian adenovirus group II splenomegaly of chickens". Avian Dis 24(3):591-594

Feichtner F, Schachner A, Berger E, Hess M (2018) Development of sensitive indirect enzyme-linked immunosorbent assays for specific detection of antibodies against fowl adenovirus serotypes 1 and 4 in chickens. Avian Pathol 47(1):73-82

Grgić H, Yang DH, Nagy E (2011) Pathogenicity and complete genome sequence of a fowl adenovirus serotype 8 isolate. Virus Res 156(12):91-97

Günes A, Marek A, Grafl B, Berger E, Hess M (2012) Real-time PCR assay for universal detection and quantitation of all five species of fowl adenoviruses (FAdV-A to FAdV-E). J Virol Methods 183(2): $147-153$

He Z, Ruan S, Zhao J, Yang H, Zhang G (2018) Recombinant fiber-2 protein-based indirect ELISA for antibody detection of fowl adenovirus serotype 4. Avian Dis 62(1):73-78

Hess M (2000) Detection and differentiation of avian adenoviruses: a review. Avian Pathol 29(3):195-206

Huang J, Tan D, Wang Y, Liu C, Xu J, Wang J (2015) Egg drop syndrome virus enters duck embryonic fibroblast cells via clathrin-mediated endocytosis. Virus Res 210:69-76

Joubert HW, Aitchison H, Maartens LH, Venter EH (2014) Molecular differentiation and pathogenicity of Aviadenoviruses isolated during an outbreak of inclusion body hepatitis in South Africa. J S Afr Vet Assoc 85(1): 1058

Kaján GL, Kecskeméti S, Harrach B, Benk"o M (2013) Molecular typing of fowl adenoviruses, isolated in Hungary recently, reveals high diversity. Vet Microbiol 167:357-363

Khawaja DA, Ahmad S, Rauf AM, Zulfiqar M, Mahmood SM, Mahmood HM (1988) Isolation of an adenovirus from hydropericardium syndrome in broiler chicks. Pak J Vet Res 1:2-17

Kim MS, Lim TH, Lee DH, Youn HN, Yuk SS, Kim BY, Choi SW, Jung $\mathrm{CH}$, Han JH, Song CS (2014) An inactivated oil-emulsion fowl Adenovirus serotype 4 vaccine provides broad cross-protection against various serotypes of fowl Adenovirus. Vaccine 32(28): 3564-3568

Li H, Wang J, Qiu L, Han Z, Liu S (2016) Fowl adenovirus species C serotype 4 is attributed to the emergence of hepatitishydropericardium syndrome in chickens in China. Infect Genet Evol 45:230-241

Lim TH, Lee HJ, Lee DH, Lee YN, Park JK, Youn HN, Kim MS, Youn HS, Lee JB, Park SY, Choi IS, Song CS (2011) Identification and virulence characterization of fowl adenoviruses in Korea. Avian Dis 55(4):554-560

Mansoor MK, Hussain I, Arshad M, Muhammad G, Hussain M, Mehmood M (2009) Molecular characterization of fowl adenovirus 
serotype 4 (FAV-4) isolate associated with fowl hydropericardiumhepatitis syndrome in Pakistan. Pak J Zool 41(4):269-276

Mase M, Nakamura K (2014) Phylogenetic analysis of fowl adenoviruses isolated from chickens with gizzard erosion in Japan. J Vet Med Sci 76:1535-1538

Matczuk AK, Niczyporuk JS, Kuczkowski M, Wozniakowski G, Nowak M, Wieliczko A (2017) Whole genome sequencing of Fowl aviadenovirus A - a causative agent of gizzard erosion and ulceration, in adult laying hens. Infect Genet Evol 48:47-53

Meng K, Yuan X, Yu J, Zhang Y, Ai W, Wang Y (2019) Identification, pathogenicity of novel fowl adenovirus serotype 4 SDJN0105 in Shandong, China and immunoprotective evaluation of the newly developed inactivated oil-emulsion FAdV-4 vaccine. Viruses 11(7)

Mittal D, Jindal N, Tiwari AK, Khokhar RS (2014) Characterization of fowl adenoviruses associated with hydropericardium syndrome and inclusion body hepatitis in broiler chickens. Virusdisease 25(1):114-119

Morshed R, Hosseini H, Langeroudi AG, Fard MHB, Charkhkar S (2017) Fowl adenoviruses D and E cause inclusion body hepatitis outbreaks in broiler and broiler breeder pullet flocks. Avian Dis 61(2):205-210

Niczyporuk JS (2016) Phylogenetic and geographic analysis of fowl adenovirus field strains isolated from poultry in Poland. Arch Virol 161(1):33-42

Niu Y, Sun Q, Zhu M, Zhao J, Zhang G, Liu X, Xiao Y, Liu S (2018) Molecular epidemiology and phylogenetic analysis of fowl adenoviruses caused hydropericardium outbreak in China during 2015. Poult Sci 97(3):803-811

Pan Q, Liu L, Gao Y, Liu C, Qi X, Zhang Y, Wang Y, Li K, Gao L, Wang X, Cui H (2017a) Characterization of a hypervirulent fowl adenovirus 4 with the novel genotype newly prevalent in China and establishment of reproduction infection model of hydropericardium syndrome in chickens. Poult Sci 96(6):1581-1588

Pan Q, Yang Y, Gao Y, Qi X, Liu C, Zhang Y, Cui H, Wang X (2017b) An inactivated novel genotype fowl adenovirus 4 protects chickens against the hydropericardium syndrome that recently emerged in China. Viruses 9(8)

Pan Q, Yang Y, Shi Z, Liu L, Gao Y, Qi X, Liu C, Zhang Y, Cui H, Wang $X(2017 c)$ Different dynamic distribution in chickens and ducks of the hypervirulent, novel genotype fowl adenovirus serotype 4 recently emerged in China. Front Microbiol 8:1005

Pan Q, Wang J, Gao Y, Cui H, Liu C, Qi X, Zhang Y, Wang X (2018) The natural large genomic deletion is unrelated to 3 the increased virulence of the novel genotype fowl Adenovirus 4 recently emerged in China. Viruses $8(8)$

Rai A, Barua S, Rai N (2005) Induction of immune response in chicken vaccinated with a plasmid dna encoding fowl adenovirus 4 hexon gene. Public Health Rep 113(6):533-534

Rajasekhar R, Roy P (2014) Recombinant hexon antigen based single serum dilution ELISA for rapid serological profiling against fowl adenovirus-4 causing hydropericardium syndrome in chickens. J Virol Methods 207:121-127

Ruan S, Zhao J, Yin X, He Z, Zhang G (2018) A subunit vaccine based on fiber-2 protein provides full protection against fowl adenovirus serotype 4 and induces quicker and stronger immune responses than an inactivated oil-emulsion vaccine. Infect Genet Evol 61:145-150

Schachner A, Marek A, Jaskulska B, Bilic I, Hess M (2014) Recombinant FAdV-4 fiber-2 protein protects chickens against hepatitishydropericardium syndrome (HHS). Vaccine 32(9):1086-1092

Shah MS, Ashraf A, Khan MI, Rahman M, Habib M, Chughtai MI, Qureshi JA (2017) Fowl adenovirus: history, emergence, biology and development of a vaccine against hydropericardium syndrome. Arch Virol 162(7):1833-1843

Shao H, Lu Y, Wang W, Li T, Zhang J, Wan Z, Liang G, Gao W, Qin A, Ye J (2019a) Two novel monoclonal antibodies against fiber-1 protein of FAdV-4 and their application in detection of FAdV-4/10. BMC Vet Res 15(1):232

Shao H, Wang P, Wang W, Zhang J, Li T, Liang G, Gao W, Qin A, Ye J (2019b) A novel monoclonal antibodies-based sandwich ELISA for detection of serotype 4 fowl adenovirus. Avian Pathol 48(3):204-208

Steer PA, Kirkpatrick NC, O'Rourke D, Noormohammadi AH (2009) Classification of fowl adenovirus serotypes by use of highresolution melting-curve analysis of the hexon gene region. J Clin Microbiol 47(2):311-321

Toro H, Prusas C, Raue R, Cerda L, Geisse C, González C, Hess M (1999) Characterization of fowl adenoviruses from outbreaks of inclusion body hepatitis/hydropericardium syndrome in Chile. Avian Dis 43(2):262-270

Vera-Hernández PF, Morales-Garzón A, Cortés-Espinosa DV, GalioteFlores A, García-Barrera LJ, Rodríguez-Galindo ET, ToscanoContreras A, Lucio-Decanini E, Absalón AE (2016) Clinicopathological characterization and genomic sequence differences observed in a highly virulent fowl Aviadenovirus serotype 4. Avian Pathol 45(1):73-81

Wang X, Tang Q, Chu Z, Wang P, Luo C, Zhang Y, Fang X, Qiu L, Dang $R$, Yang Z (2018) Immune protection efficacy of FAdV-4 surface proteins fiber-1, fiber-2, hexon and penton base. Virus Res 245:1-6

Ye J, Liang G, Zhang J, Wang W, Song N, Wang P, Zheng W, Xie Q, Shao H, Wan Z, Wang C, Chen H, Gao W, Qin A (2016) Outbreaks of serotype 4 fowl adenovirus with novel genotype, China. Emerg Microbes Infect 5(5):e50

Yuan X, Wang Y, Meng K, Zhang Y, Xu H, Ai W (2019) LAMP real-time turbidity detection for fowl adenovirus. BMC Vet Res 15(1):256

Zhai X, Mei X, Wu X, Zuo L, Zhou L, Tian Y, Han X, Yang X, Wang H (2019) A loop-mediated isothermal amplification coupling with a lateral flow dipstick for rapid and specific detection of fowl adenovirus serotype-4. J Virol Methods 270:79-86

Zhao J, Zhong Q, Zhao Y, Hu YX, Zhang GZ (2015) Pathogenicity and complete genome characterization of fowl adenoviruses isolated from chickens associated with inclusion body hepatitis and hydropericardium syndrome in China. PLoS One 10(7):e0133073

Publisher's note Springer Nature remains neutral with regard to jurisdictional claims in published maps and institutional affiliations. 